\title{
CEACAM1 and molecular signaling pathways to expand the liver transplant donor pool
}

\author{
Samer Tohme and David A. Geller \\ Division of Hepatobiliary and Pancreatic Surgery, Department of Surgery, University of Pittsburgh, Pittsburgh, Pennsylvania, USA.
}

\begin{abstract}
Organ shortage continues to limit the lives of patients who require liver transplantation. While extending criteria for liver organs provides a needed resource, tissue damage from prolonged ischemic injury can result in early allograft dysfunction and consequent rejection. In this issue of the $J \mathrm{Cl}$, Nakamura et al. used a mouse transplantation model with prolonged ex vivo cold storage to explore liver graft protection. The authors found that liver grafts with absent carcinoembryonic antigen-related cell adhesion molecule 1 (CEACAM1) exhibited increased ischemia-reperfusion injury inflammation and decreased function in wild-type recipients. The authors went on to correlate CEACAM1 levels with postreperfusion damage in human liver transplant recipients. Notably, this study identified a potential biomarker for liver transplant donor graft quality.
\end{abstract}

\section{Organ shortage remains} the Achilles heel of liver transplantation

Liver transplants can save the lives of patients suffering with end-stage liver disease or liver tumors. Indications for liver transplantation are expanding, and transplant outcomes continue to improve with one-year graft and patient survival now around $90 \%$ (1). However, organ shortage remains the Achilles heel of liver transplantation. Liver transplantation, as is true with other organs, has been hampered by the lack of available donors in relation to the number of people waiting. As a result, three people die every day in the United States while waiting for a liver transplant. There are currently 12,643 patients on the national waiting list. In 2019, 8896 liver transplants were performed in the United States and $20 \%$ of waitlisted candidates were removed from the list because they became too sick, had tumor progression, or died (2). Thus, the same question remains - where do we find more organs?
Organ shortage has prompted the use of extended criteria donor organs from older, steatotic donors without a heartbeat, or organs that have been subjected to prolonged periods of storage. These livers, referred to as marginal, are particularly susceptible to ischemia-reperfusion injury (IRI) (3). Warm IRI, which is initiated by hepatocellular damage, develops during liver transplantation surgery or during various forms of shock or trauma, and might lead to liver or even multiorgan failure (4). Cold IRI occurs during ex vivo preservation and is usually coupled with warm IRI during liver transplantation surgery. Both warm and cold IRIs are characterized by an initial local inflammatory response characterized by the production of various danger-associated molecular patterns (DAMPs) such as HMGB1, cytokines (IL-2, IL-6), chemokines (CXCL8, CXCL10), and reactive oxygen species (ROS). This is accompanied by innate immune activation with the activation of liver Kupffer cells and neutrophils (5-7). This inflammato-

\section{Related Article: p. 2689}

Conflict of interest: The authors have declared that no conflict of interest exists.

ry progression is continued by infiltrating lymphocytes and/or monocytes (5). Cold IRI from preservation injury also affects liver sinusoidal endothelial cells (LSECs) and leads to increased endothelin (ET) and thromboxane A2 (TXA2) and decreased nitrous oxide (NO). Cold IRI also favors an early and massive $\mathrm{T}$ cell influx into the liver grafts (8). The crosstalk between the innate and adaptive immune responses triggered by IRI readily converts the liver into an inflammatory organ (Figure 1). IRI contributes to the donor organ shortage (as organs affected by cold IRI may be deemed too damaged for transplantation), poor early graft function and primary nonfunction, and is a major risk factor for both acute and chronic rejection (9). Despite its clear clinical importance, the mechanisms that account for liver IRI are only partly understood and remain an understudied area in transplantation.

\section{Protective role of CEACAM1 during cold ischemia}

In this issue of the JCI, Nakamura et al. used a clinically relevant mouse model of orthotopic liver transplantation (OLT) with prolonged ex vivo cold storage (10). The researchers found that liver grafts that lacked carcinoembryonic antigen-related cell adhesion molecule 1 (CEACAM1) exhibited increased IR inflammation and decreased function in wild-type recipients. This inflammatory phenotype was accompanied by release of high-mobility group box 1 (HMGB1), leukocyte trafficking, and a proinflammatory genetic profile. CEACAM1 hepatoprotective role paralleled the inhibition of apoptosis signal-regulating kinase 1 (ASK1)/p-p38 pathways and cell death. Having identified the pathways related to the protective role of CEACAM during cold ischemia, the authors moved to correlate CEACAM1 levels and postreperfusion damage in human liver transplant recipients. Notably, the authors found that low CEACAM1 expression in the donor livers prior to implantation independently 


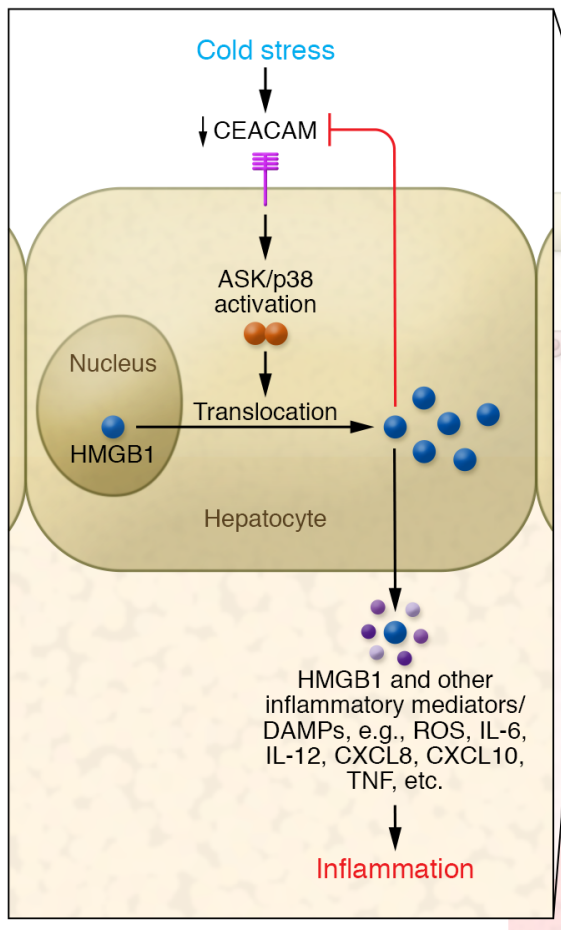

Liver sinusoid
IRI

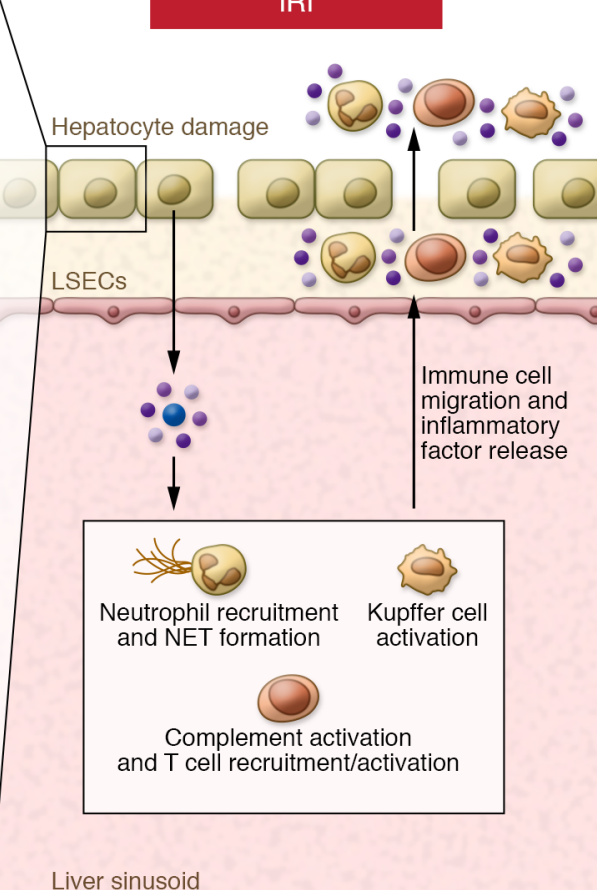

Graft rejection

predicted early isograft dysfunction. This carefully performed mechanistic study shows a unique role for CEACAM1, serving in a protective manner during I/R injury. A strength of the study is the bedside correlate in human liver transplant biopsies, which identifies CEACAM1 as a potential biomarker for liver transplant graft quality.

\section{Future research}

Several unanswered questions remain. What is the contributing hepatoprotective effect of CEACAM1 expressed on the immune cell infiltrate? What drives CEACAM1 over- and underexpression in the liver, and can this upstream mechanism be manipulated to confer protection to the organ? Although the authors present human data that broadly support the results from the mouse models, further research is needed to determine whether targeting the ASK1/p-p38 pathway or other implicated pathways has the potential to improve graft outcomes after liver transplantation (Figure 1).

With expanding indications for liver transplantation and the shortage of organs, future research should focus on identifying alternative ways to find transplantable organs. Some experimental concepts, such as xenotransplantation and organ manufacturing, are promising concepts, but their clinical feasibility is likely far in the future. Machine perfusion of marginal livers is increasingly used to assess function and perfusion. With an increase in number of livers being transplanted from donors after cardiac death, ischemic cholangiopathy is a concern for transplant centers in terms of patient outcomes as well as transplant center graft and patient survival metrics (11). Ex vivo interventions to target cold IRI are crucial to save organs and improve outcomes after transplantation. Targeting IRI at the bedside can occur in preconditioning or postconditioning transplant settings. Preconditioning applies to pharmacological interventions in the donor or graft before implantation or reperfusion, whereas postconditioning refers to the time of reperfusion in transplant recipients. Although an array of agents are protective against liver IRI in animal models, the few that have been tested in randomized liver transplantation controlled trials showed limited success (5).

The liver transplant field is ever changing and evolving. As one problem
Figure 1. Model of the molecular mechanism for warm and cold IRI. Cold stress decreases CEACAM1 expression, which activates ASK/ p38 and subsequent HMGB1 translocation from the nucleus to the cytoplasm to further block CEACAM1 and continue the inflammatory progression. Both cold and warm IRI activate the innate immune system, including liver Kupffer cells and neutrophils. The adaptive immune system is also triggered, eliciting complement activation and T cell activation. Involved inflammatory cells produce cytokines and chemokines, and generate ROS, further contributing to graft dysfunction. is conquered, another takes its place. It is unlikely that the need for liver transplantation will go away, or that the organ supply will suddenly get better. Therefore, it is necessary to have a better understanding of liver IRI to provide the rationale for much needed agents to fill the current therapeutic gaps to improve graft function. It is critical to identify therapeutic targets at the bench and to bring them to the bedside in order to improve donor organ quality, save lives, benefit patient outcomes, and ultimately enhance the success of liver transplantation.

Address correspondence to: David A. Geller, MUH 7S Liver Cancer Center, 3459 Fifth Avenue, Pittsburgh, Pennsylvania 15213, USA. Phone: 412.692.2001; Email: gellerda@upmc.edu.

1. Hughes CB, Humar A. Liver transplantation: current and future [published online January 17, 2020]. Abdom Radiol (NY). https://doi. org/10.1007/s00261-019-02357-w.

2. US Department of Health and Human Services. Organ Procurement and Transplantation Network Database. http://optn.transplant.hrsa.gov. Accessed March 25, 2020.

3. Attia M, Silva MA, Mirza DF. The marginal liver donor--an update. Transpl Int. 2008;21(8):713-724. 
4. Kaczorowski DJ, Tsung A, Billiar TR. Innate immune mechanisms in ischemia/reperfusion. Front Biosci (Elite Ed). 2009;1:91-98.

5. Zhai Y, Petrowsky H, Hong JC, Busuttil RW, Kupiec-Weglinski JW. Ischaemia-reperfusion injury in liver transplantation--from bench to bedside. Nat Rev Gastroenterol Hepatol. 2013;10(2):79-89.

6. Huang H, et al. Damage-associated molecular pattern-activated neutrophil extracellular trap exacerbates sterile inflammatory liver injury. Hepatology. 2015;62(2):600-614.
7. Montalvo-Jave EE, Escalante-Tattersfield T, Ortega-Salgado JA, Piña E, Geller DA. Factors in the pathophysiology of the liver ischemia-reperfusion injury. J Surg Res. 2008;147(1):153-159.

8. Shen XD, et al. Disruption of Type-I IFN pathway ameliorates preservation damage in mouse orthotopic liver transplantation via HO-1 dependent mechanism. Am J Transplant. 2012;12(7):1730-1739.

9. Lentsch AB, Kato A, Yoshidome H, McMasters KM, Edwards MJ. Inflammatory mechanisms and therapeutic strategies for warm hepatic ischemia/reperfusion injury. Hepatology. 2000;32(2):169-173.

10. Nakamura K, et al. Hepatic CEACAM1 expression indicates donor liver quality and prevents early transplantation injury. J Clin Invest. 2020;130(5):2689-2704.

11. Fung JJ, Eghtesad B, Patel-Tom K. Using livers from donation after cardiac death donors--a proposal to protect the true Achilles heel. Liver Transpl. 2007;13(12):1633-1636. 